\title{
Field Theory Conjecture for Loop-Erased Random Walks
}

\author{
Andrei A. Fedorenko • Pierre Le Doussal • \\ Kay Jörg Wiese
}

Received: 21 May 2008 / Accepted: 23 October 2008 / Published online: 6 November 2008

(C) Springer Science+Business Media, LLC 2008

\begin{abstract}
We give evidence that the functional renormalization group (FRG), developed to study disordered systems, may provide a field theoretic description for the loop-erased random walk (LERW), allowing to compute its fractal dimension in a systematic expansion in $\varepsilon=4-d$. Up to two loop, the FRG agrees with rigorous bounds, correctly reproduces the leading logarithmic corrections at the upper critical dimension $d=4$, and compares well with numerical studies. We obtain the universal subleading logarithmic correction in $d=4$, which can be used as a further test of the conjecture.
\end{abstract}

Keywords Loop-erased random walks · Sandpiles · Functional renormalization group

The loop-erased random walk (LERW) was introduced by Lawler [1] as an alternative to the self-avoiding walk (SAW), which is relevant in polymer physics. On the lattice, the LERW is defined as the trajectory of a random walk in which any loop is erased as soon as it is formed. As the SAW, the LERW has no self-intersections, but is more tractable mathematically. It has been proven that the LERW has a scaling limit in all dimensions [2-7]. The number of steps (or time) $t$ it takes to reach the distance $L$ scales as $t_{L} \sim L^{z}$, where $z$ is the fractal dimension of LERW. In $d=2$ this scaling limit is conformally invariant and described $[8,9]$ by the stochastic Loewner evolution $\mathrm{SLE}_{2}$. Though the LERW and SAW belong to different

\footnotetext{
A.A. Fedorenko $(\bowtie) \cdot$ P. Le Doussal · K.J. Wiese

CNRS-Laboratoire de Physique Théorique de l'Ecole Normale Supérieure, 24 rue Lhomond, 75231

Paris Cedex, France

e-mail: andrey.fedorenko@ens-lyon.fr

P. Le Doussal

e-mail: 1edou@1pt.ens.fr

K.J. Wiese

e-mail: wiese@lpt.ens.fr

Present address:

A.A. Fedorenko

CNRS UMR 5672—Laboratoire de Physique de l'Ecole Normale Supérieure de Lyon, 46 Allée d'Italie, 69007 Lyon, France
} 
universality classes, the LERW itself has received significant attention due to applications in combinatorics, self-organized criticality (SOC), and, more recently, conformal field theory and SLE. The LERW can be viewed as a special case of the Laplacian random walk [10] and can be mapped [4] to the problem of uniform spanning trees (UST): Chemical (i.e. shortest) paths on UST obey LERW statistics. It is proven that the upper critical dimension is $d_{\mathrm{uc}}=4$, the same as for the SAW, since for $d>4$ the traces of two random walks do almost surely not intersect. Hence for $d>4$ the fractal dimension of the LERW is that of a simple random walk, $z=2$. It was proven a while ago [11] that for $d<4$ it is bounded from above by the Flory estimate for the SAW exponent:

$$
R^{2} \sim t^{2 / z}, \quad z<\frac{d+2}{3}=2-\frac{\varepsilon}{3},
$$

where $R=\left\langle R(t)^{2}\right\rangle^{1 / 2}$ is the radius of gyration, and we have introduced $\varepsilon=4-d$. The mapping to UST and equivalently to the $q$-state Potts model at $q \rightarrow 0$ was used in $d=2$ to predict [4] $z_{\mathrm{LERW}}(d=2)=\frac{5}{4}$, later proved in [12]. It is a particular case, for $\kappa=2$, of the fractal dimension $d_{\mathrm{f}}=1+\frac{\kappa}{8}$ of the trace of $\mathrm{SLE}_{\kappa}$ (a simple curve for $\kappa<4$ ). Another connection in $d=2$ is to the $O(N)$ loop model with $N=-2$, both corresponding to a conformal field theory (CFT) with central charge $c=-2$. The leading logarithmic corrections at the upper critical dimension $d=4$ where obtained by Lawler [5] who proved that:

$$
R^{2} \sim t(\ln t)^{1 / 3} .
$$

In $d=3$ the value of $z_{\text {LERW }}$ is known only from numerics. The most precise estimate was obtained by Agrawal and Dhar [13],

$$
z_{\text {LERW }}(d=3)=1.6183 \pm 0.0004 \text {, }
$$

improving on the previous numerical result [14] $z=1.623 \pm 0.011$.

Contrary to the SAW, which is described by the $O(N)$ model at $N=0$, there seems to be at present no field-theoretic approach to compute the LERW exponent in a dimensional expansion around $d=4$. This is surprising, especially when compared with the recent progress in CFT descriptions in $d=2$. In this short paper we propose a field theoretic description for the LERW, based on the Functional RG, a method developed to study disordered systems. We build on a connection proposed a while ago between the depinning transition of periodic elastic systems, also called charge density waves (CDW) in random media and sandpile models. The correspondence, on which we detail below, is indirect, through a chain of related models: From LERW to UST to sandpiles to CDW-depinning and finally to functional renormalization group (FRG) field theory. Some of the connections are not rigorous. At the end we show that the FRG passes all the tests of presently known results for LERW. In particular, it reproduces the correct leading logarithmic corrections in $d=4$ given by (2), and makes a prediction for the subleading logarithmic correction which we hope will be tested in the near future.

We now present briefly the intermediate models. The Bak-Tang-Wiesenfeld (BTW) sandpile model was proposed as a prototype for driven dissipative systems exhibiting SOC [15]. It is defined on the $d$-dimensional hyper-cubic lattice with $L^{d}$ sites. The configuration at time $t$ is given by the integer number of grains $h(x, t)$ at site $x$. The site $x$ is unstable if $h(x, t)>2 d$ in which case it relaxes according to the toppling rule

$$
\begin{aligned}
& h(x, t+1)=h(x, t)-2 d, \\
& h(y, t+1)=h(y, t)+1,
\end{aligned}
$$


where $y$ denotes all the $2 d$ nearest neighbors of site $x$. The neighbor sites may then become unstable and the toppling continue. The order of topplings is irrelevant, thus the notion of "Abelian" sandpile, which allows to use e.g. parallel dynamics. The process continues until no unstable site remains, i.e. the avalanche ends. This is achieved through grains that leave the system at the boundary. To ensure a steady state one drives the system by adding a grain to a randomly chosen site $x$ after each avalanche. Stable configurations are either transient, or recurrent in which case they appear in the steady state with equal probability [16].

Using the burning algorithm a one-to-one correspondence is set up between the recurrent configurations of sandpile models and spanning trees on the same lattice plus a sink $x_{0}$ (into which fall all grains leaving the system through the boundary) [17]. These spanning trees are connected sets of edges touching all lattice sites with no loops, and chosen with uniform probability (UST). Due to the Abelian property any avalanche initiated at an arbitrary site $x_{i}$ can be decomposed into successive toppling waves, such that during one wave each site topples at most once [18]. Each wave is characterized by a two-root spanning tree on the extended lattice with connected $x_{i}$ and $x_{0}$. The subtree with the root at $x_{i}$ spans the sites toppled by the wave while the subtree with the root at $x_{0}$ connects the sites not affected by the wave. This spanning tree gives a unique (chemical) path between two toppled sites separated by the distance $L$. The number of edges along this path is nothing but the number of update steps between these two toppling events, i.e. the time $t$ between them. Thus the time $t$ and length $L$ in the sandpile dynamics are related by the fractal dimension of the chemical path along a spanning tree [19], which is nothing but a LERW.

Narayan and Middleton proposed that the charge density wave (CDW) near the depinning transition can be viewed to some extent as a BTW sandpile model [20, 21]. The configuration of a $d$-dimensional elastic object, such as a CDW, pinned by random impurities is parameterized by a displacement field $u_{x t}, x \in \mathbb{R}^{d}$. Here we assume that the distortions of the object caused by disorder are continuous. For CDWs $u_{x t}$ is the local phase divided by $2 \pi$. In the continuous limit, the dynamics is described by the over-damped equation of motion

$$
\eta \partial_{t} u_{x t}=\nabla^{2} u_{x t}+F\left(x, u_{x t}\right)+f
$$

where $\eta$ is the friction, $f$ the driving force, e.g. the external electric field for CDW, and $F$ the impurity pinning force. The latter is taken to be Gaussian with zero mean and correlator

$$
\overline{F(x, u) F\left(x^{\prime}, u^{\prime}\right)}=\Delta\left(u-u^{\prime}\right) \delta^{d}\left(x-x^{\prime}\right),
$$

where $\Delta(u)$ is an even periodic function (chosen here with period 1). For CDWs $F(x, u)$ is often written as

$$
F(x, u)=\alpha(x) Y[u-\gamma(x)]
$$

with $|Y(u)| \leq 1$ being a periodic function with period 1. The strength $\alpha(x)$ of impurities and their selected phase $\gamma(x) \in[0,1)$ are taken to be random and uncorrelated in space. Both forms (6) and (7) of disorder correlations are equivalent at least in the vicinity of the so-called depinning transition. The system driven by a homogenous force $f$ undergoes a depinning transition at a critical force $f_{c}$ : below the transition $f<f_{c}$ the system is pinned while above it, it exhibits a never-ending motion. This dynamic transition is reminiscent of an ordinary second-order phase transition with the velocity playing the role of the order parameter: $v \sim\left(f-f_{c}\right)^{\beta}$ and enjoys a similar universality, i.e. independence of the scaling behavior on the microscopic details. The correlation length $\xi$ defined through the velocityvelocity correlation function diverges at the transition as $\xi \sim\left(f-f_{c}\right)^{-v}$; the length and time 
scales of fluctuations are related by the dynamic critical exponent $z: t \sim x^{z}$. Discretizing space and time, one can rewrite (5) near the depinning transition in the form of the automaton model (4) with $h(x, t)$ playing the role of a coarse-grained curvature of the elastic object. For a pinning potential with narrow and steep wells the configuration of the CDW can be encoded by integer variables $m(x, t)=u(x, t)-\gamma(x)$. Upon time and space discretization $m(x, t)$ evolves according to the following dynamic rule [20]

$$
m(x, t+1)=m(x, t)+\Theta\left\{f+\nabla^{2}[\gamma(x)+m(x, t)]+\alpha(x)\right\}
$$

where $\Theta(x)$ is the Heaviside step function and $\nabla^{2}$ is the discrete Laplace operator. In terms of a coarse-grained curvature defined as

$$
h(x)=\sum_{y}[m(y)-m(x)]+\operatorname{int}\left[f+\nabla^{2} \gamma(x)+\alpha(x)\right],
$$

the dynamics rule (8) can be expressed in the form of the toppling rule (4) with an inessential difference that the stability threshold now is $h(x)=0$ not $h(x)=2 d$. Increasing $f$ toward the critical force is equivalent to increasing $h$ by 1 at a random site. Thus both models, the original BTW model and the discretized version of (5), are driven by adding grains, $h(x, t+1)=h(x, t)+1$. In the CDW model grains are added with a cycle restriction: a second grain can be added to a particular site only if all other sites have received a grain. The ordering of sites in the cycle is provided by the value of the quenched random term $\nabla^{2} \gamma(x)+\alpha(x)$ in (9). While this difference between the pinned at threshold CDW and BTW may seem inconsequential for large systems, it illustrates that the mapping is presently not rigorous; moreover an ad-hoc discretization was used. Nevertheless it is supported by numerics [20]. It strongly suggests that the dynamic exponent $z$ describing the depinning transition of a $d$-dimensional CDW coincides with the fractal dimension of LERW in $d$ dimensions.

The field theory which describes the depinning transition is based on the Functional RG $[22,23]$. Applying the Martin-Siggia-Rose formalism to (5) and averaging over disorder with the help of (6) one arrives at the action

$$
S=\int_{x t} i \hat{u}_{x t}\left(\eta \partial_{t}-\nabla^{2}\right) u_{x t}-\int_{x t} i \hat{u}_{x t} f_{x t}-\frac{1}{2} \int_{x t t^{\prime}} i \hat{u}_{x t} i \hat{u}_{x t^{\prime}} \Delta\left(u_{x t}-u_{x t^{\prime}}\right),
$$

where the response field $\hat{u}_{x t}$ has been introduced. We argue that the field theory defined by (10) also describes the LERW. The dynamic exponent of model (10) at criticality is nothing but the fractal dimension of LERW. We also expect that more properties of LERWs are encoded in the fixed point $\Delta^{*}(u)$ since it controls the large-scale behavior of the model. At this stage, however, we only extract the relation between time $t$ and space $L$, more work is needed to elucidate relations to other observables. Power counting shows that the upper critical dimension of the model is $d_{\mathrm{uc}}=4$. The peculiarity of the problem is that all derivatives of $\Delta(u)$ at the origin $u=0$ become relevant operators below $d_{\mathrm{uc}}=4$. Thus, instead of a finite number of coupling constants we have to deal with a whole coupling function $\Delta(u)$. To extract the scaling behavior one has to study the flow of the renormalized function $\Delta(u)$ under changing the infrared cutoff towards infinity. Recent progress has shown that full consistency requires a 2-loop study. The corresponding flow equations to 2-loop order read $[24,25]$

$$
\partial_{l} \Delta(u)=\varepsilon \Delta(u)-\frac{1}{2}\left[(\Delta(u)-\Delta(0))^{2}\right]^{\prime \prime}
$$




$$
\begin{array}{r}
+\frac{1}{2}\left[(\Delta(u)-\Delta(0)) \Delta^{\prime}(u)^{2}\right]^{\prime \prime}+\frac{1}{2}\left[\Delta^{\prime}\left(0^{+}\right)\right]^{2} \Delta^{\prime \prime}(u), \\
\partial_{l} \ln \eta=-\Delta^{\prime \prime}(0)+\Delta^{\prime \prime}(0)^{2}+\Delta^{\prime \prime \prime}\left(0^{+}\right) \Delta^{\prime}\left(0^{+}\right)\left[\frac{3}{2}-\ln 2\right],
\end{array}
$$

where $l=\ln L$, and $L$ the infrared cutoff, e.g. the size of the system. Below $d=4$ the flow equation (11) has a fixed point (FP) solution $\Delta^{*}(u)$ with a cusp at the origin: $\Delta^{* \prime}\left(0^{+}\right) \neq 0$. Taking into account that $t \sim \eta_{l} L^{2}$ and that in the vicinity of the FP $\eta_{l}$ scales with $L$ according to (12), one finds that $t \sim L^{z}$, and to 2-loop order the exponent $z$ is given by [24, 25]:

$$
z=2-\frac{\varepsilon}{3}-\frac{\varepsilon^{2}}{9}+O\left(\varepsilon^{3}\right)
$$

Comparison with the exact bound (1) is encouraging since the 2-loop correction has the correct sign. To estimate values of $z$ in $d=2,3$ we compute different Padé approximants $[n / m]$ to $(13)$. In $d=2$ we obtain $z(d=2)=1.23 \pm 0.22$, consistent with the presumed exact value $\frac{5}{4}$ but of poor accuracy ${ }^{1}$ due to the large expansion parameter $\varepsilon=2$. Table 1 contains the approximants $[n / m]$ in $d=3$. To improve the accuracy we construct the higher order Padé approximants with $n+m=3$ imposing that $z(d=2)=\frac{5}{4}$ for $\varepsilon=2$. The average and root-mean-square of all approximants with $n+m=3$ yields

$$
z=1.614 \pm 0.011 \text {. }
$$

The same procedure based on one loop only produces $z=1.638 \pm 0.012$. The value (14) is our best 2-loop FRG prediction for $d=3$ and is in fairly good agreement with the numerical result (3).

The field theory is most predictive at the upper critical dimension, where it yields exact results. For FRG some were obtained previously (see e.g. [26-28]). Here we compute the logarithmic corrections to the dynamics which yield a prediction for the LERW in $d=4$. For $\varepsilon=0$ one shows from (11) that the (periodic) disorder correlator approaches the FP solution $\Delta^{*}(u)=0$ as follows (for $\left.0 \leq u \leq 1\right)$ :

$$
\Delta_{l}(u)=\left[\frac{1}{6 l}+\frac{\ln l}{9 l^{2}}\right]\left[\frac{1}{6}-u(1-u)\right]+O\left(\frac{1}{l^{2}}\right) .
$$

Substituting (15) into (12) we obtain

$$
\ln \frac{\eta_{l}}{\eta_{0}}=-\frac{1}{3} \ln l+\frac{2 \ln l}{9 l}+O\left(\frac{1}{l}\right) .
$$

Table 1 Padé approximants for $z$ in $d=3$

\begin{tabular}{lllll}
\hline$[n / m]$ & $m=0$ & $m=1$ & $m=2$ & $m=3$ \\
\hline$n=0$ & 2 & 1.71429 & 1.6 & 1.61074 \\
$n=1$ & 1.66667 & 1.5 & 1.61194 & \\
$n=2$ & 1.55556 & 1.63158 & & \\
$n=3$ & 1.60069 & & & \\
\hline
\end{tabular}

\footnotetext{
${ }^{1}$ We used the average and root mean squared of the set $z_{[1 / 0]}=4 / 3, z_{[0 / 1]}=3 / 2, z_{[2 / 0]}=8 / 9, z_{[0 / 2]}=6 / 5$. We excluded approximant $[1 / 1]$ which accidentally vanishes.
} 
Renormalizing the relation $t \sim \eta_{l} L^{2}$ up to scale $L$ we arrive at

$$
t \sim L^{2}(\ln L)^{-1 / 3}\left[1+\frac{2 \ln \ln L}{9 \ln L}+O\left(\frac{1}{\ln L}\right)\right],
$$

which can be rewritten as

$$
L^{2} \sim t(\ln t)^{1 / 3}\left[1-\frac{\ln \ln t}{3 \ln t}+O\left(\frac{1}{\ln t}\right)\right] .
$$

The scale $L^{2}$ can be taken as $R^{2}$, the radius of gyration. We note that the leading order of (18) coincides with the result (2) of Lawler. Here we obtain the universal subleading correction.

The prediction (18) could be tested numerically, very much as for the corresponding prediction [29] for the SAW in $d=4$, checked in [30]; there subleading corrections are necessary to properly fit the numerical data at any feasible chain length. To this purpose, it is useful to note that, as for the SAW, there is only a single non-universal constant in the correction term in the parenthesis in (18), $c / \ln t$. It can e.g. be put to zero by a proper choice of $t_{0}$, setting $t \rightarrow t / t_{0}$ (in which case further corrections are $1 / \ln ^{2} t$ and determined by 3 - and higher-loop terms, not considered here). For comparison, one similarly finds, from the 2-loop $\beta$-function [31] of the $O(N)$ model in $d=4$ that $L^{2} \sim t(\ln t)^{a_{N}}\left(1-b_{N} \frac{\ln \ln t}{\ln t}\right)$ with $a_{N}=(N+2) /(N+8)$ and $b_{N}=(N+2)\left(68+8 N-N^{2}\right) /(N+8)^{3}$ from which the Duplantier values for the SAW [29], $a_{0}=1 / 4$ and $b_{0}=17 / 64$ are retrieved at $N=0$. Note that no value of $N$ can account for (18), thus a representation of LERW via the $O(N)$ field theory, if feasible at all, would at least require a more complicated operator correspondance.

Note finally that the exponent $\tau$ for the avalanche-size distribution ${ }^{2}$ was recently computed to one loop within FRG [32, 33]. In the sandpile literature no controlled calculation exists for the corresponding exponent, usually called $\tau_{s}$, but the formula $\tau_{s}(d)=2-2 / d$ leading to $\tau_{s}=4 / 3$ in $d=3$ has been conjectured [13] from scaling arguments. The FRG was found to agree to $O(\epsilon)$ with this formula, assuming $\tau=\tau_{s}$. Evaluation of 2-loop corrections is in progress as a further test of the conjecture and of the relations between sandpile models and depinning.

To conclude, it may appear surprising that LERW be related to a field theory based on functional RG, where one would expect a "simpler", more conventional, field theory based on a single relevant coupling constant, as for the SAW. One clue may be that for periodic systems (CDW) the FRG posesses a stable submanifold with only two coupling constants, $\Delta(u)=a+b u(1-u)($ for $0 \leq u \leq 1)$, which contains the leading critical behavior. From (11) one obtains $[24,25,33]$ the spectrum of convergence to the fixed point ${ }^{3}$ as $1 /(\ln L)^{1+\alpha_{n}}$ in $d=4$ and $L^{-\omega_{n}}$ with $\omega_{n}=\alpha_{n} \epsilon+\beta_{n} \epsilon^{2}+O\left(\epsilon^{2}\right)$ for $d<4 ; \alpha_{n}=\frac{1}{3}(3+n)(1+2 n)$, $\beta_{n}=-\frac{1}{9}(n+2)(2 n+1)(2 n+3)$. The values $n=1,2, \ldots$ correspond to the convergence to the submanifold, and $n=0$ to the convergence inside it. Convergence to the submanifold is fast, with leading eigenvalue $1+\alpha_{1}=5$. Fast convergence was also found in numerics [34] where the FRG function $\Delta(u)$ in (11) was directly measured in $d=1,2,3$. The conjecture raises many other interesting issues to be explored, such as the possibility to predict other LERW observables and corrections to scaling, the role of other universality classes for CDW and interface depinning, the connections to fermionic field theory (known for UST, see e.g. [35], and conjectured for the FRG [28]). Work is in progress in these directions.

\footnotetext{
${ }^{2}$ The probability to have an avalanche of size $s$ is proportional to $s^{-\tau}$, with a cutoff for large and small avalanches.

${ }^{3}$ Apart from the trivial uniform mode of eigenvalue $\epsilon$.
} 
Acknowledgements We acknowledge support from the Agence Nationale de la Recherche under program 05-BLAN-0099-01 and from the European Commission under contract No. MIF1-CT-2005-021897 (AAF).

\section{References}

1. Lawler, G.F.: A self avoiding walk. Duke Math. J. 47, 655-693 (1980)

2. Duplantier, B.: Loop-erased self-avoiding walks in two dimensions: exact critical exponents and winding numbers. Physica A 191, 516-522 (1992)

3. Kozma, G.: The scaling limit of loop-erased random walk in three dimensions. Acta Math. 199, 29-152 (2007). arXiv:math/0508344

4. Majumdar, S.N.: Exact fractal dimension of the loop-erased self-avoiding walk in two dimensions. Phys. Rev. Lett. 68, 2329-2331 (1992)

5. Lawler, G.F.: The logarithmic correction for loop-erased walk in four dimensions. In: Proceedings of the Conference in Honor of Jean-Pierre Kahane (Orsay, 1993). J. Fourier Anal. Appl. (Special issue) 347-362 (1995)

6. Lawler, G.F.: Intersections of Random Walks. Birkhäuser Boston, Cambridge (1991)

7. Lawler, G.F.: Loop-erased random walk. In: Perplexing Problems in Probability. Progress in Probability vol. 44, pp. 197-217. Birkhäuser Boston, Cambridge (1999)

8. Schramm, O.: Scaling limits of loop-erased random walks and uniform spanning trees. Isr. J. Math. 118, 221-288 (2000). arXiv:math/9904022

9. Lawler, G.F., Schramm, O., Werner, W.: Conformal invariance of planar loop-erased random walks and uniform spanning trees. Ann. Probab. 32, 939-996 (2004). arXiv:math/0112234

10. Lawler, G.F.: Loop-erased self-avoiding random walk and the Laplacian random walk. J. Phys. A 20, 4565-4568 (1987)

11. Lawler, G.F.: Loop-erased self-avoiding random walk in two and three dimensions. J. Stat. Phys. 50, 91-108 (1988)

12. Kenyon, R.: The asymptotic distribution of the discrete Laplacian. Acta Math. 185, 239-286 (2000)

13. Agrawal, H., Dhar, D.: Distribution of sizes of erased loops of loop-erased random walks in two and three dimensions. Phys. Rev. E 63, 056115 (2001)

14. Guttman, A.J., Bursill, R.J.: Critical exponent for the loop erased self-avoiding walk by Monte Carlo methods. J. Stat. Phys. 59, 1-9 (1990). arXiv:math-ph/0011042

15. Bak, P., Tang, C., Wiesenfeld, K.: Self-organized criticality. An explanation of the 1/f noise. Phys. Rev. Lett. 59, 381-384 (1987)

16. Dhar, D.: Self-organized critical state of sandpile automaton models. Phys. Rev. Lett. 64, 1613-1616 (1990)

17. Majumdar, S.N., Dhar, D.: Equivalence between the Abelian sandpile model and the $q \rightarrow 0$ limit of the Potts model. Physica A 185, 129-145 (1992)

18. Priezzhev, V.B., Ktitarev, D.V., Ivashkevich, E.V.: Formation of avalanches and critical exponents in an Abelian sandpile model. Phys. Rev. Lett. 76, 2093 (1996)

19. Ktitarev, D.V., Lübeck, S., Grassberger, P., Priezzhev, V.B.: Scaling of waves in the Bak-Tang-Wiesenfeld sandpile model. Phys. Rev. E 61, 81 (2000)

20. Narayan, O., Middleton, A.A.: Avalanches and the renormalization group for pinned charge-density waves. Phys. Rev. B 49, 244-256 (1994)

21. Alava, M.: Scaling in self-organized criticality from interface depinning? J. Phys. Condens. Mater 14, 2353-2360 (2002)

22. Narayan, O., Fisher, D.S.: Threshold critical dynamics of driven interfaces in random media. Phys. Rev. B 48, 7030-7042 (1993)

23. Nattermann, T., Stepanow, S., Tang, L.H., Leschhorn, H.: Dynamics of interface depinning in a disordered medium. J. Phys. II (France) 2, 1483-1488 (1992)

24. Chauve, P., Le Doussal, P., Wiese, K.J.: Renormalization of pinned elastic systems: how does it work beyond one loop? Phys. Rev. Lett. 86, 1785-1788 (2001)

25. Le Doussal, P., Wiese, K.J., Chauve, P.: Two-loop functional renormalization group theory of the depinning transition. Phys. Rev. B 66, 174201 (2002)

26. Chitra, R., Giamarchi, T., Le Doussal, P.: Disordered periodic systems at the upper critical dimension. Phys. Rev. B 59, 4058-4065 (1999). arXiv:cond-mat/9809300

27. Fedorenko, A.A., Stepanow, S.: Depinning transition at the upper critical dimension. Phys. Rev. E 67, 057104 (2003)

28. Le Doussal, P., Wiese, K.J.: Higher correlations. universal distributions, and finite size scaling in the field theory of depinning. Phys. Rev. E 68, 046118 (2003). arXiv:cond-mat/0301465 
29. Duplantier, B.: Polymer chains in four dimensions. Nucl. Phys. B 275, 319-355 (1986)

30. Grassberger, P., Hegger, R., Schäfer, L.: Self-avoiding walks in 4 dimensions-logarithmic corrections. J. Phys. A 27, 7265-7282 (1994)

31. Zinn-Justin, J.: Quantum Field Theory and Critical Phenomena. Oxford University Press, London (1989)

32. Le Doussal, P., Middleton, A.A., Wiese, K.J.: Statistics of static avalanches in a random pinning landscape. arXiv:0803.1142 (2008). Le Doussal P. and Wiese K.J.: In preparation

33. Fedorenko, A.A., Le Doussal, P., Wiese, K.J.: Statistics of avalanches at the depinning transition. In preparation

34. Middleton, A.A., Le Doussal, P., Wiese, K.J.: Measuring functional renormalization group fixed-point functions for pinned manifolds. Phys. Rev. Lett. 98, 155701 (2007). arXiv:cond-mat/0606160

35. Read, N.: Exponents and bounds for uniform spanning trees in d dimensions. Phys. Rev. E 70, 027103 (2004) 